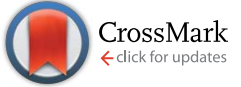

Cite this: RSC Adv., 2017, 7, 13858

Received 3rd January 2017

Accepted 21st February 2017

DOI: $10.1039 / c 7 r a 00086 c$

rsc.li/rsc-advances

\section{Mutational analysis to identify the residues essential for the acetyltransferase activity of GlmU in Bacillus subtilis}

\begin{abstract}
Meng Wang, Minhua Huang, Huawei Gu, Shan Li, Yi Ma* and Jufang Wang*
The novel and attractive antimicrobial drug target, glucosamine-1-phosphate acetyltransferase $/ \mathrm{N}$ acetylglucosamine-1-phosphate uridyltransferase (GlmU), is a bifunctional enzyme that catalyzes two sequential steps in the biosynthesis of UDP-GlcNAc, essential for both lipopolysaccharide and peptidoglycan in Gram positive and Gram negative bacteria. Glucosamine-1-phosphate acetyltransferase catalyzes the formation of $\mathrm{N}$-acetylglucosamine-1-phosphate and $\mathrm{N}$-acetylglucosamine-1-phosphate uridylyltransferase catalyzes the formation of UDP-GlcNAc. In this study, glmU genes from Escherichia coli and Bacillus subtilis were cloned and the corresponding proteins were produced in an E. coli BL21 (DE3) expression system. The kinetic parameters and reaction conditions, such as initial reaction rate, optimal temperature and $\mathrm{pH}$, and the effect of $\mathrm{Mg}^{2+}$ ion concentration of $\mathrm{GlmU}$ enzymes from $E$. coli (Ec-GlmU) and B. subtilis (Bs-GlmU) were detected. Compared to Ec-GlmU, Bs-GlmU exhibits much lower glucosamine-1-phosphate acetyltransferase activity. Based on the in silico results of a virtual amino acid mutation and molecular docking of Bs-GlmU, five mutants were successfully constructed to verify the predicted alterations of acetyltransferase activity. The result of point mutations indicated that the $\mathrm{C} 405$ and $\mathrm{A} 380$ residues played key role in catalytic mechanism of acetyltransferase of Bs-GlmU providing new insight into the reaction mechanism of $\mathrm{GlmU}$ enzymes and supporting the further development of antimicrobial drugs.
\end{abstract}

\section{Introduction}

Uridine $5^{\prime}$-diphospho $N$-acetylglucosamine (UDP-GIcNAc), an acetylated nucleotide sugar, plays an important role in the biochemistry of all living organisms..$^{1-3} \mathrm{As}$ an essential substrate to synthesize many biomolecules, including lipopolysaccharide, peptidoglycan and oligosaccharides, UDP-GIcNAc has high potency to exploit pharmaceuticals and functional materials. ${ }^{4,5}$ However, due to the lack of an efficient method to synthesize this product, UDP-GlcNAc is in great demand and its price is notoriously high. ${ }^{6,7}$ Infection caused by pathogenic bacteria has become a serious threat to public health in recent years. A sharp rise in bacteria resistant to antibiotics has emerged as a serious issue which renders drugs ineffective for fighting against bacterial infection. ${ }^{8,9}$ Few new antibiotics to combat Grampositive organisms and no new drugs for Gram-negative pathogens have become available for clinical research or in the pharmaceutical market for last 40 years. ${ }^{10-12}$

Recent research has suggested that enzymes involved in cell wall biosynthesis could be useful targets for developing new antibiotics. ${ }^{13,14}$ Peptidoglycan is a major component of the cell

School of Bioscience and Bioengineering, South China University of Technology, 510006 Guangzhou, China. E-mail: bimayikobe@scut.edu.cn; jufwang@scut.edu.cn; Fax: +86-20-39383034; +86-20-39380626; Tel: +86-20-39383034; +86-20-39380626 wall that provides structural strength and rigidity and is essential for survival and growth of the bacterium. ${ }^{15-17}$ Peptidoglycan chains are made up of a repeating $N$-acetylglucosamine (NAG) and $N$-acetylmuramic acid (NAM) units, which are cross linked by peptide bridges. ${ }^{18}$ Several reactions in different cytoplasmic and membranes steps are involved in the biosynthesis of peptidoglycan. Three enzymes, glutamine fructose-6-phosphate transferase (GlmS), phosphoglucosamine mutase (GlmM) and glucosamine-1-phosphate acetyltransferase/ $N$-acetylglucosamine-1-phosphate uridyl transferase (GlmU), participate in the synthesis of UDPGlcNAc, which is the main intermediate in the formation of peptidoglycan and lipid A. ${ }^{16,19,20}$ Both peptidoglycan and lipid A are necessary for cell viability and perturbing UDP-GlcNAc synthesis which catalyzed by GlmU have grave effects on the fitness of the bacteria. ${ }^{21,22}$

GlmU catalyzes two consecutive reactions: the first is an acetyltransferase reaction with the help of acetyl coenzyme A (Ac-CoA) to convert $\alpha$-D-glucosamine-1-phosphate (GlcN1P) to $N$ acetyl $\alpha$-D-glucosamine-1-phosphate (GlcNAc1P) and produces the by-product coenzyme A (CoA). The second step is a uridyltransferase reaction converting GlcNAc1P to UDP-GlcNAc using UTP as a uridyl group donor and releasing pyrophosphate (PPi) ${ }^{23-25}$ The first step is widespread in bacteria enzymes but is missing in humans, whereas the uridyltransferase step is 
present in enzymes of both bacteria and humans. ${ }^{26,27}$ The acetyltransferase domain could be a suitable target for developing nontoxic antibacterial inhibitors. ${ }^{28}$

The study of Ec-GlmU have confirmed that the N-terminal portion of the enzyme contains the uridylyltransferase domain and the C-terminus functions as an acetyltransferase. ${ }^{29,30}$ The crystal structure of Ec-GlmU with multiple substrates revealed that the 2-amino group of GlcN-1- $\mathrm{PO}_{4}$ is positioned in proximity to AcCoA to facilitate direct thioester attack by a ternary complex mechanism. ${ }^{31,32}$ The cell wall structure of Gram-positive bacteria is quite different to that of Gram-negative bacteria. There is urgent need to explore Grampositive bacteria such as B. subtilis since understanding BsGlmU will provide important information for developing novel inhibitors. However, there are few articles pertaining to Bs-GlmU. The GcaD protein in B. subtilis is the similar to GlmU with uridyltransferase activity but no acetyltransferase activity. ${ }^{33,34}$ In this study, Bs-GlmU is recombinant expressed and characterized and the structure model of Bs-GlmU is obtained. Based on the in silico results of virtual amino acid mutation and molecular docking of Bs-GlmU, five mutants are constructed to investigate the mechanism and key amino acid residues of acetyltransferase in Bs-GlmU, providing a new template for understanding the structure and function of GlmU in Gram-positive bacteria. Our results will support further GlmU research and facilitate high-throughput inhibitor screening.

\section{Materials and methods}

\subsection{Materials}

Reagents related to $E$. coli and B. subtilis glmU gene cloning and site-directed mutagenesis were purchased from TakaRa Biotechnology Co., Ltd (Dalian, China). Competent cells E. coli DH5 $\alpha$ and BL21 (DE3) were obtained from TIANGEN Biotech (Beijing, China). The Ni-NTA His-binding column from QAIGEN (Dusseldorf, Germany) was used for protein purification. The chemicals used for enzyme assays were purchased from Sigma-
Aldrich Chemical Co. (St. Louis, MO, USA). All chemical reagents used in this study were analytical grade.

\subsection{Nucleic acid manipulations}

Genome DNA from E. coli and B. subtilis were prepared using a genome extraction kit (Sangon, Shanghai, China) as described in the manufacturer's handbook. The GlmU genes of E. coli (EcglmU) and B. subtilis (Bs-glmU) were amplified by PCR reaction using Prime STAR HS DNA Polymerase (TaKaRa, Dalian, China) from the genome DNA of E. coli and B. Subtilis strain respectively. The forward primer used for Ec-glmU was 5' CGGGATCC ATGTTGAATAATGCTATGAGCGTAGTGA-3' (BamH I restriction site underlined), and the reverse primer for EcglmU was 5'-CCGCTCGAG CTTTTTCTTTACCGGACGACGCCAG-3' (Xho I restriction site underlined). The forward primer used for Bs-glmU was 5'-CGGGATCC ATGGATAAGCGG TTTGCAGTTGTTT-3' (BamH I restriction site underlined), and the reverse primer for Bs-glmU was $5^{\prime}$ CCGCTCGAG TTTTTTATGAATATTTTTCACATAATCGTC-3' (Xho I restriction site underlined). Ec-glmU was amplified in $50 \mu \mathrm{L}$ of reaction buffer by initial denaturation temperature at $98{ }^{\circ} \mathrm{C}$ for 2 min followed by 32 cycles of $98{ }^{\circ} \mathrm{C}$ for $10 \mathrm{~s}, 71^{\circ} \mathrm{C}$ for $15 \mathrm{~s}$, and $72{ }^{\circ} \mathrm{C}$ for $90 \mathrm{~s}$ with a final $10 \mathrm{~min}$ extension at $72{ }^{\circ} \mathrm{C}$. Bs-glmU was amplified in $50 \mu \mathrm{L}$ of reaction buffer by initial denaturation temperature at $98{ }^{\circ} \mathrm{C}$ for $2 \mathrm{~min}$ followed by 32 cycles of $98{ }^{\circ} \mathrm{C}$ for $10 \mathrm{~s}, 65{ }^{\circ} \mathrm{C}$ for $15 \mathrm{~s}$, and $72{ }^{\circ} \mathrm{C}$ for $90 \mathrm{~s}$ with a final $10 \mathrm{~min}$ extension at $72{ }^{\circ} \mathrm{C}$. The Ec-glmU and Bs-glmU genes were digested with BamH I and Xho I (Fermentas of Thermo Scientific, Pittsburgh, PA), then ligated with the double digested pET21a vector (Novagen, Wisconsin, USA) yielding the plasmids pET-Ec-glmU and pET-Bs-glmU, respectively. Competent $E$. coli DH5 $\alpha$ cells (Invitrogen, CA, USA) were used as the host for cloning. Transformed cells were spread on Luria-Bertani agar plates containing $100 \mathrm{ng} \mu \mathrm{L}^{-1}$ of ampicillin and incubated at $37{ }^{\circ} \mathrm{C}$ overnight. The positive clones were identified by colony PCR and confirmed by DNA sequence analysis. Sequence similarity searches were performed with the BLAST program (http:// www.ncbi.nlm.nih.gov/BLAST/).

Table 1 Oligonucleotide primers used to construct mutant plasmids ${ }^{a}$

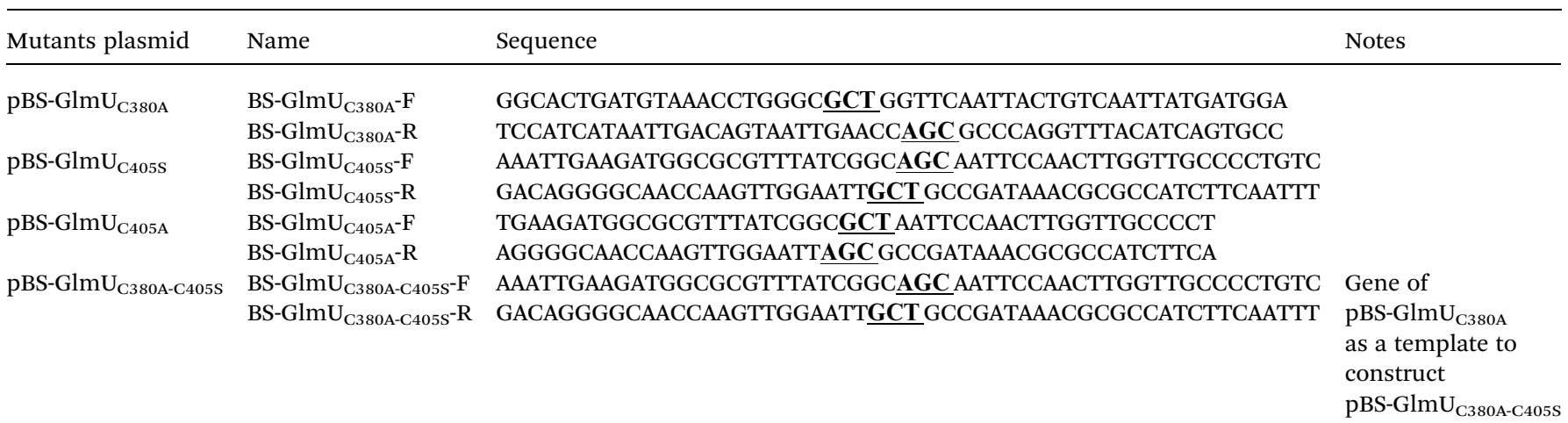

pBS-GlmU $U_{\mathrm{V} 386 \mathrm{C}-\mathrm{C} 405 \mathrm{~S}} \quad$ BS-GlmU $\mathrm{V}_{\mathrm{V} 386 \mathrm{C}-\mathrm{C} 405 \mathrm{~s}^{-\mathrm{F}}}$ AAACCTGGGCTGCGGTTCAATTACTTGCAATTATGATGGAAAGA BS-GlmU U ${ }_{\mathrm{V} 386 \mathrm{C}-\mathrm{C} 405 \mathrm{~s}^{-} \mathrm{R}}$ TCTTTCCATCATAATTGCA AGTAATTGAACCGCA GCCCAGGTTT

${ }^{a}$ Mutated sites are marked in bold and underlined. 


\subsection{Expression and purification of Ec-GlmU and Bs-GlmU}

Plasmids pET-Ec-glmU and pET-Bs-glmU were transformed into E. coli BL21 (DE3) (Novagen, Madison, WI, USA). The recombinant bacteria were induced to express recombinant Ec-GlmU and Bs-GlmU by adding isopropyl- $\beta$-D-thiogalactopyranoside (IPTG) at a final concentration of $1 \mathrm{mM}$ to a culture with $\mathrm{OD}_{600}$ of approximately $0.6-0.8$ and incubated at $37^{\circ} \mathrm{C}$ for $3 \mathrm{~h}$. Cell cultures $(1 \mathrm{~L})$ were separated by centrifugation at $5000 \times \mathrm{g}$ for $20 \mathrm{~min}$. The cell pellets were re-suspended in $35 \mathrm{~mL}$ of binding buffer (20 mM Tris-HCl, $500 \mathrm{mM} \mathrm{NaCl,} \mathrm{20 \%} \mathrm{Glycerin,} 50 \mathrm{mM}$ imidazole, $\mathrm{pH} 8.0)$ and lysis with 450 sonication pulses $(400 \mathrm{~W}$, $3 \mathrm{~s}$ with a $5 \mathrm{~s}$ interval) cooled in an ice water bath. ${ }^{35-37}$ The suspension was centrifuged $\left(11000 \times g\right.$ at $4{ }^{\circ} \mathrm{C}$ for $\left.30 \mathrm{~min}\right)$, the supernatant passed through a $0.22 \mu \mathrm{m}$ filter and applied to a HiTrap ${ }^{\mathrm{TM}}$ Chelating HP column (GE Healthcare, Piscataway, NJ, USA). The Ec-GlmU and Bs-GlmU were purified using the standard nickel affinity chromatography procedure with elution buffer (20 mM Tris-HCl, $500 \mathrm{mM} \mathrm{NaCl,} \mathrm{20 \%} \mathrm{glycerol,} 500 \mathrm{mM}$ imidazole, $\mathrm{pH}$ 8.0). The purity of the proteins was evaluated using SDS-PAGE and the concentrations were determined by a BCA Protein Assay Kit (Sangon, Shanghai, China). Elution fractions of the containing proteins of interest were held in storage buffer $(20 \mathrm{mM}$ Tris- $\mathrm{HCl}, 50 \mathrm{mM} \mathrm{NaCl}, 20 \%$ glycerin, $\mathrm{pH}$ 8.0) at $-80^{\circ} \mathrm{C}$.

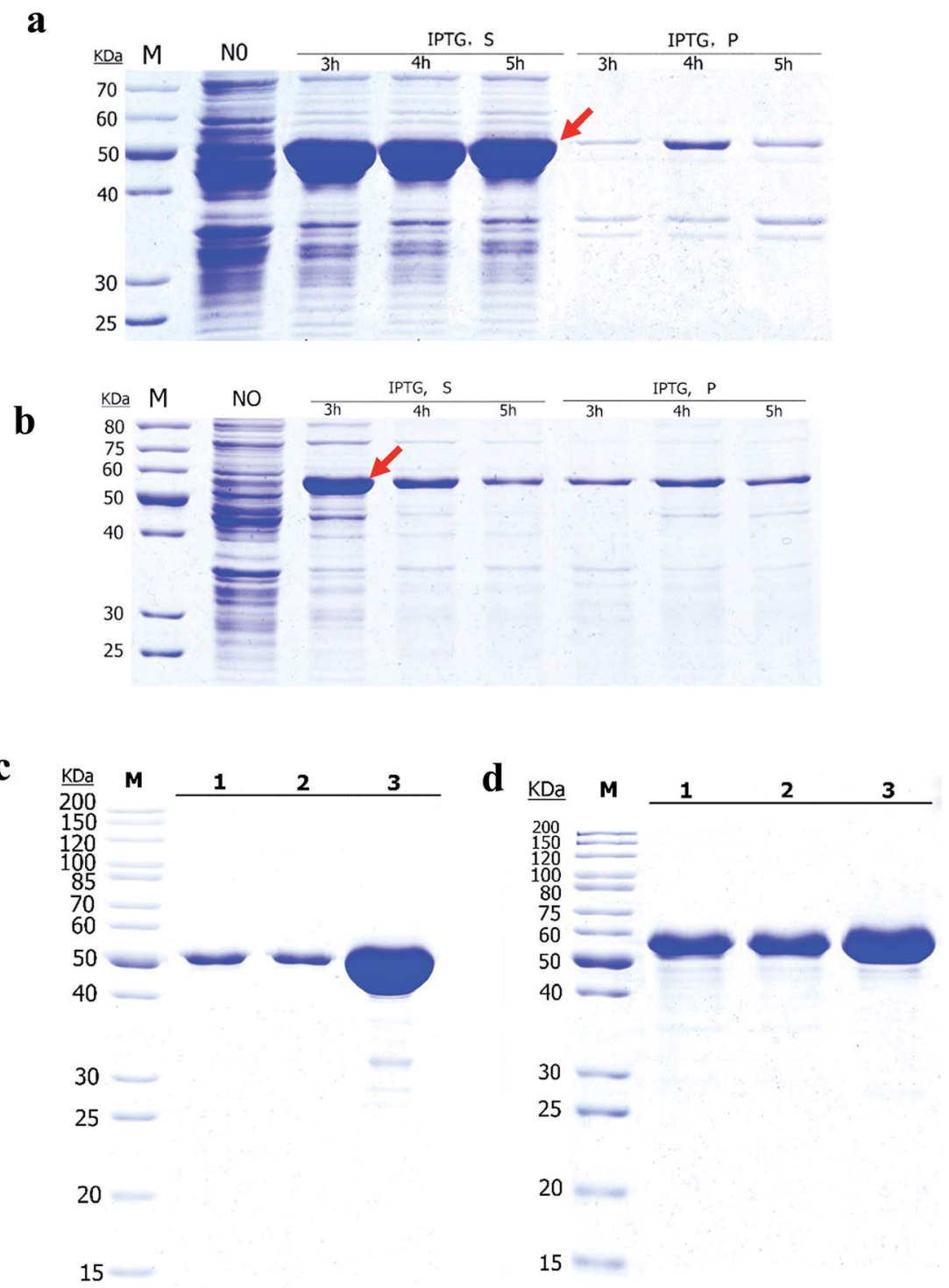

Fig. 1 Expression and purification of Bs-GlmU and Ec-GlmU in BL21 (DE3) cells. SDS-PAGE analysis of Ec-GlmU (a and c) and Bs-GlmU (b and d). A total of $1 \mathrm{mM}$ IPTG was added into the cells when $\mathrm{OD}_{600}$ reach to $0.4-0.6$. "NO" indicates that the cells were not induced with IPTG. Cells were induced with IPTG for 3, 4, or $5 \mathrm{~h}$ respectively. "IPTG S" means supernatant fractions of induced cells after sonication; "IPTG P" indicates the insoluble pellets of induced cells after sonication. "1, 2, 3" in (c) and (d) indicates different concentrations of Ec-GlmU and Bs-GlmU. Positions corresponding to Ec-GlmU and Bs-GlmU are marked by arrows. Optimal conditions of $1 \mathrm{mM}$ IPTG and $3 \mathrm{~h}$ expression were used for the purified Ec-GlmU, Bs-GlmU. M, protein marker. 


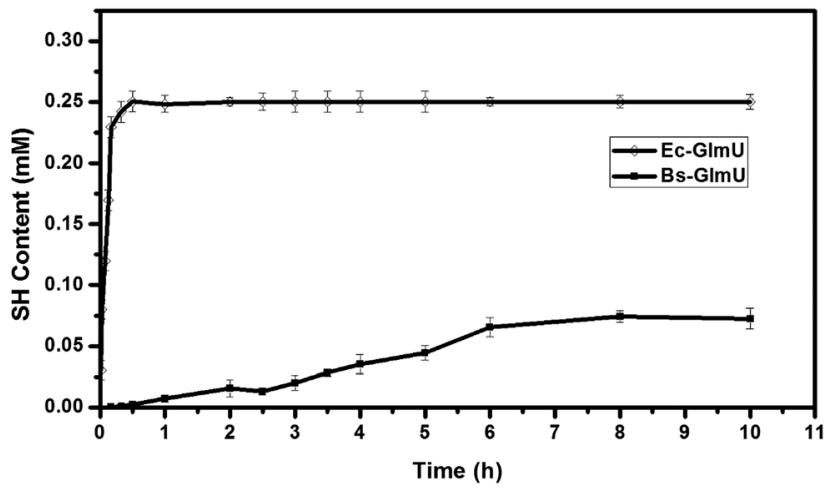

Fig. 2 Characterization and analysis of acetyltransferase activities between Ec-GlmU and Bs-GlmU. A colorimetric assay coupled with DTNB was taken for the detection of acetyltransferase activities. The acetyltransferase activities of Ec-GlmU, open diamonds; Bs-GlmU, filled square were measured in triplicate.

\subsection{Enzymatic activity}

The glucosamine-1-phosphate acetyltransferase activities of the wild-type and mutant GlmU were measured by a colorimetric microplate assay with 5, 5'-dithiobis (2-nitrobenzoic acid, DTNB). ${ }^{38,39}$ The $50 \mu \mathrm{L}$ reaction mixture (50 mM Tris- $\mathrm{HCl}, \mathrm{pH}$ 8.0, $5 \mathrm{mM} \mathrm{MgCl}_{2}$, $0.5 \mathrm{mM}$ GlcN-1-P, $0.5 \mathrm{mM}$ AcCoA, and $0.5 \mathrm{ng}$ purified GlmU protein) was incubated in a 96 well microtiter plate at $37{ }^{\circ} \mathrm{C}$ for different time gradients from $1 \mathrm{~min}$ to 10 hours. The reaction was terminated by addition of $50 \mu \mathrm{L}$ stop solution (50 mM Tris- $\mathrm{HCl}, 6.4 \mathrm{M}$ guanidine hydrochloride, $\mathrm{pH}$ 8.0) and the mixture was incubated for $10 \mathrm{~min}$ at $37^{\circ} \mathrm{C}$ with 50 $\mu \mathrm{L}$ Ellman's reagent solution (0.2 mM DTNB in buffer [50 $\mathrm{mM}$ Tris-HCl, $1 \mathrm{mM}$ EDTA, $\mathrm{pH} 8.0]){ }^{35}$ The production of $\mathrm{TNB}^{2-}$, generated from the reaction of CoA-SH and DTNB, was monitored at a wavelength of $412 \mathrm{~nm}$. A blank control with GlcN-1-P and AcCoA with inactive GlmU was used to correct the trial errors generated from the $-\mathrm{SH}$ group of GlmU protein.

\subsection{Molecular modeling and docking}

The 3D model of full-length Bs-GlmU was obtained by the homology modeling method on I-TASSER server. ${ }^{40}$ Five prokaryotic GlmU from E. coli K-12 (PDBID: 2OI5), M. tuberculosis Rv37 (PDBID: 3DK5-A), Streptococcus pneumonia (PDBID: 1HM9-A), Yersinia pestis (PDBID: 3FWW-A) and Haemophilus influenza (PDBID: 2VOH-A) were selected as structural models to improve the accuracy of modeling. The initial AcCoA binding mode to Bs-GlmU was modeled through alignment of the EcGlmU and Bs-GlmU structures. Clashes between Bs-GlmU and AcCoA were removed by 2000 step energy minimization using NAMD2 program with CHARMM22 force field. ${ }^{41}$ AcCoA force
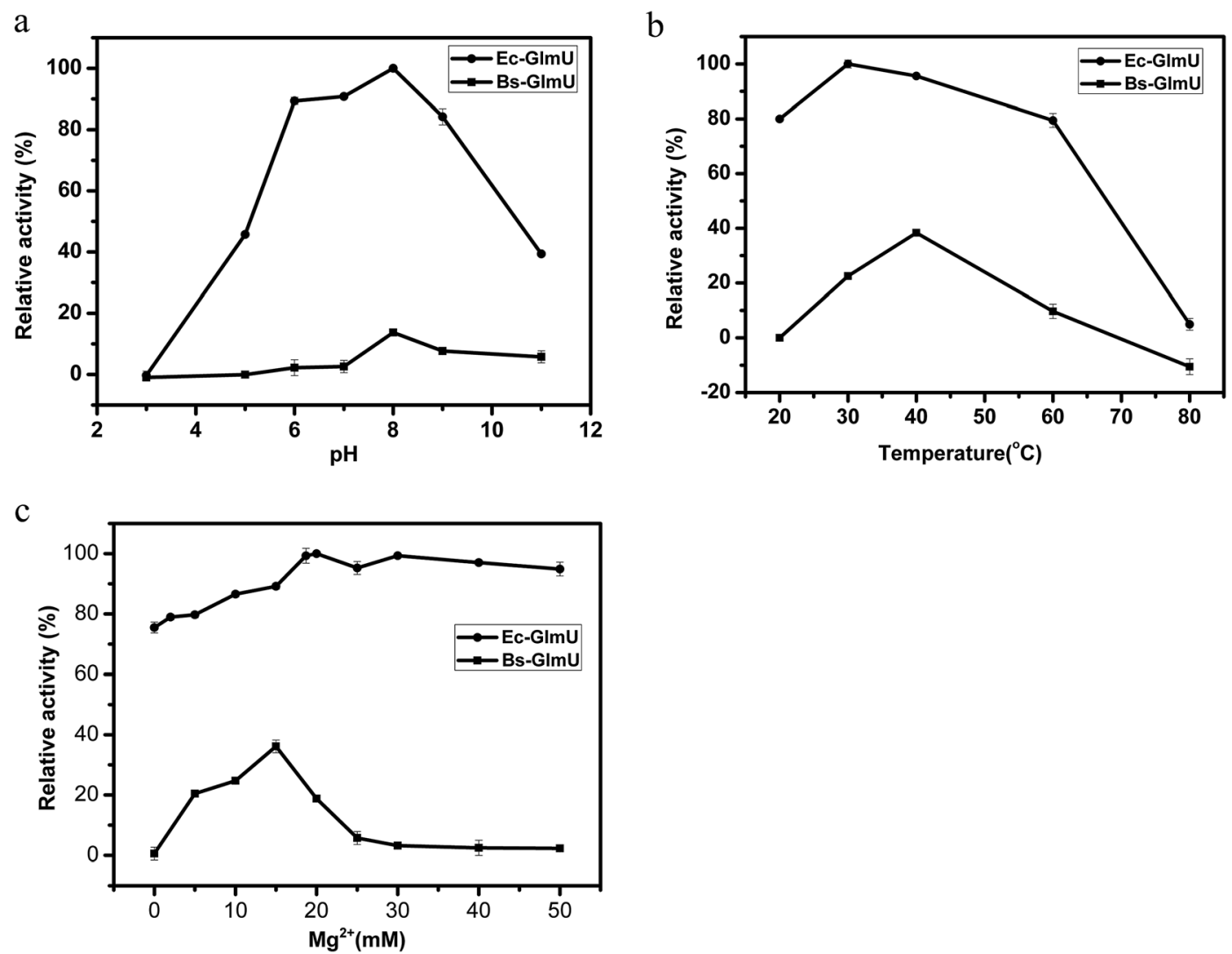

Fig. 3 Determination of optimal conditions for Ec-GlmU and Bs-GlmU. (a) The effect of pH (3-11) on Ec-GlmU (solid round) and Bs-GlmU (filled square) acetyl transferase activities. $\mathrm{pH}$ buffers with final concentration of $50 \mathrm{mM}$ were as follows: citric acid-sodium citrate ( $\mathrm{pH} 3.0)$; $\mathrm{NaH}_{2} \mathrm{PO}_{4}-$ $\mathrm{Na}_{2} \mathrm{HPO}_{4}\left(\mathrm{pH}\right.$ 5.0-6.0); Tris- $\mathrm{HCl}(\mathrm{pH} 7.0-9.0) ; \mathrm{NH}_{4} \mathrm{Cl}-\mathrm{NH}_{3} \mathrm{H}_{2} \mathrm{O}$ (pH 11). (b) Different incubation temperatures on the acetyl transferase activities of Ec-GlmU and Bs-GlmU. (c) Different concentrations of $\mathrm{Mg}^{2+}$. 
field parameter was assigned by the CHARMM General Force field (CGenFF) program on ParamChem server. ${ }^{42}$ The mutated structures were modeled by visual molecular dynamics, ${ }^{43}$ and 2000 step energy minimization applied to refine the structures. All structural representations were drawn by visual molecular Dynamics Software.

\subsection{In vitro mutagenesis}

Multiple protein sequences, including target protein sequences, were aligned and analyzed. The amino acid sites selected for mutation are shown in the Table 1 . In recent years, the method called Restriction Free (RF) cloning has been widely applied to insert desired DNA bases in mutation studies. ${ }^{44,45}$ This restriction free cloning PCR method was applied to construct the mutated genes from the wild-type Bs-glmU gene template using the primer pairs shown in Table 1. For double mutation in plasmid, this method was used with DNA sequence analysis for site mutations one by one to obtain the required plasmid. The GlmU proteins were expressed with a C-terminal $6 \times$ His tag to allow protein purification that was provided by pET-21a $(+)$ fused with an expression tag.

\subsection{Kinetic studies of GlmU acetyltransferase}

The initial velocity of bifunctional GlmU was closely associated with both the reaction time and concentration curves. ${ }^{46}$ Conditions to optimize the initial velocity of GlmU were investigated in the ranges of $\mathrm{pH} 3-\mathbf{1 1}$ at $20-80{ }^{\circ} \mathrm{C}$ for temperature, and $0-25 \mathrm{mM}$ for $\mathrm{Mg}^{2+}$ concentration. The steady-state kinetic parameters $K_{\mathrm{m}}$ and $V_{\max }$ for two substrates (GlcN1P and AcCoA) were determined at various concentrations ( 0.05 to $5 \mathrm{mM})$ of one substrate maintaining the other substrate at fixed value $(0.5$ $\mathrm{mM}$ ) for $10 \mathrm{~min}$ under optimal reaction conditions. The fixed value $(0.5 \mathrm{mM})$ has been saturated for all GlmU enzymes by the pre-experiment results (data not show). All measurements were performed in triplicate and the data were analyzed by GraphPad software program (GraphPad Software, Inc. CA, USA).

\section{Results and discussion}

\subsection{Expression and purification of Ec-G $\operatorname{lm} U$ and Bs-GlmU in E. coli}

The Ec-GlmU and Bs-GlmU were expressed as C-terminal $6 \times$ His tag fusion proteins using pET21a expression vectors in $E$. coli BL21 (DE3). The CA tag was fused at the N-terminal of Ec-GlmU and Bs-GlmU to achieve high yield. Ec-GlmU and Bs-GlmU have shown protein bands corresponding to about $52 \mathrm{kDa}$ in SDSPAGE (Fig. 1a and b). Both Ec-GlmU and Bs-GlmU were expressed in the soluble fraction with a yield of about $85 \%$ after $3 \mathrm{~h}$ induction with IPTG (Fig. 1a and b). No more target proteins was produced in longer induction time. The proteins then were purified by affinity chromatography and both purity reached $95 \%$ detected by SDS-PAGE gel (Fig. 1c and d).

\subsection{Characterization and analysis of acetyltransferase activities of Ec-GlmU and Bs-GlmU}

Elman's Reagent (DTNB) reacted with materials containing a$\mathrm{SH}$ group and produces yellow $\mathrm{TNB}^{2-}$, which has particularly absorption at $412 \mathrm{~nm} .{ }^{38}$ This assay with high sensitivity was

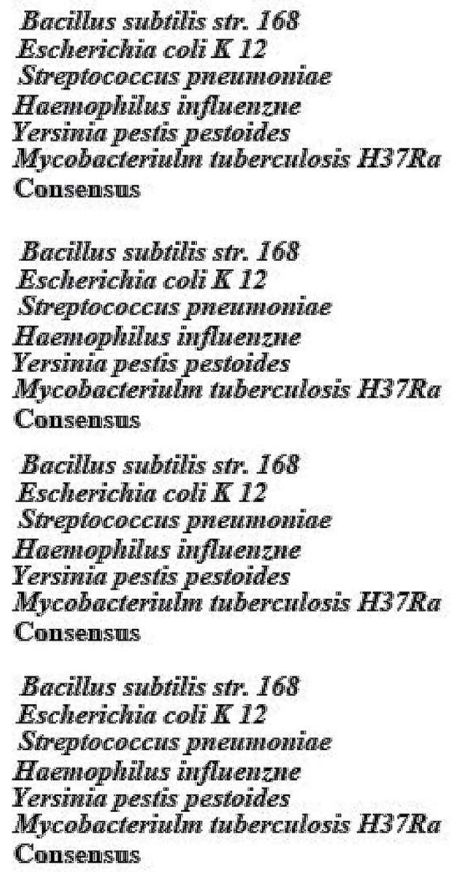

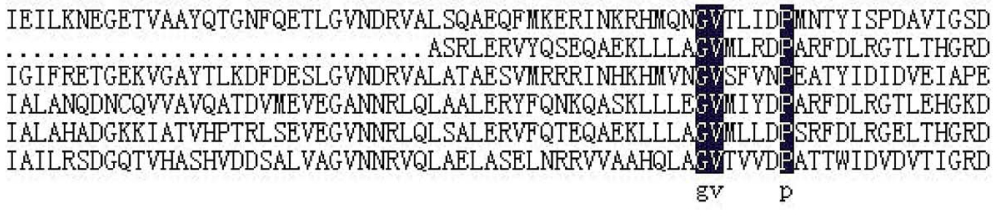
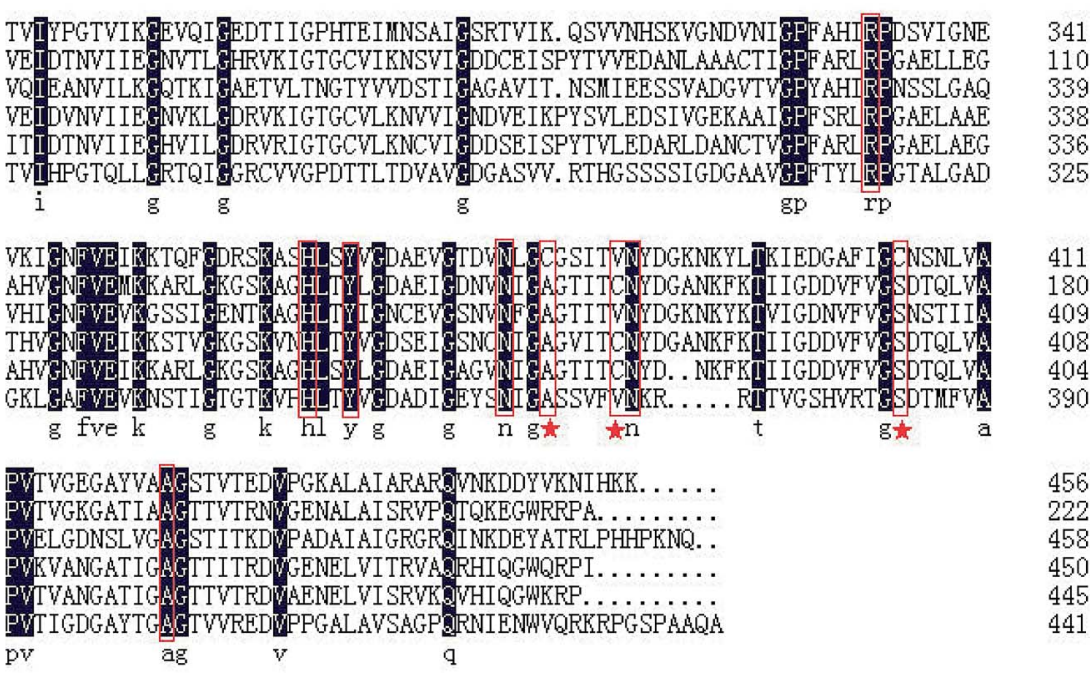

Fig. 4 Sequence alignment of Bs-GlmU and other GlmU amino acid sequence. Five GlmU from the following microorganism were selected: Escherichia coli K12, Mycobacterium tuberculosis H37Ra, Haemophilus influenzae, Yersinia pestis pesticides, Streptococcus pneumoniae. The residues in red box are those involved in acetyl transferase activities. The residues marked with asterisk are the key amino acid which was mutated in this study. 


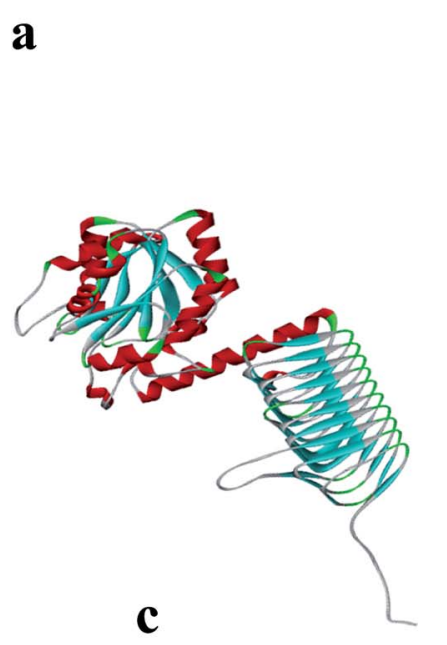

b

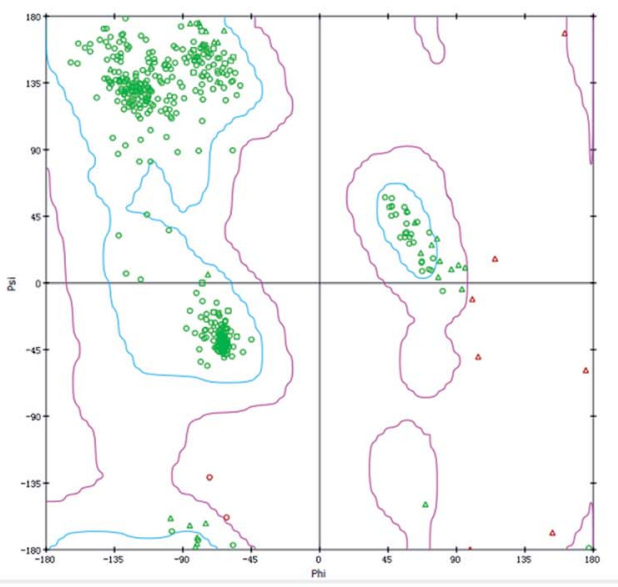

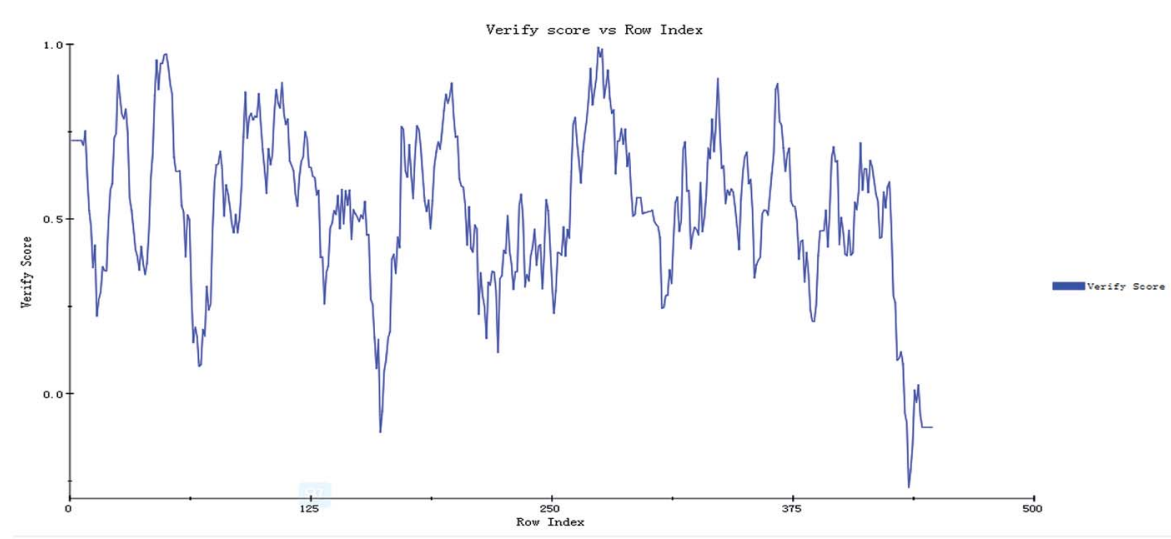

Fig. 5 Model of the monomeric structure of Bs-GlmU. (a) The modeled three-dimensional structure of Bs-GlmU. (b) The Ramachandran statistics of the simulated Bs-GlmU structure, showing the residues in the optimum region (blue), the allowed region (magenta), and the disallowed region (red triangle). (c) The structure of Bs-GlmU that was verified using Profile-3D.

used to detect the -SH produced from AcCoA by Ec-GlmU and Bs-GlmU acetyltransferase catalysis. ${ }^{38}$ The reaction with EcGlmU reaches equilibrium in $30 \mathrm{~min}$ however Bs-GlmU need 6 hours. Furthermore, the total $-\mathrm{SH}$ produced from AcCoA by Ec-GlmU was 2.5 fold than that produced by Bs-GlmU (Fig. 2). The acetyltransferase activities of Ec-GlmU was similar to other reports. ${ }^{47}$ Also, the GlmU acetyltransferase activity was tested from pH 3 to 11 and the optimal pH for both enzymes was 8.0 (Fig. 3a). The GlmU acetyltransferase activity was observed from 20 to $80{ }^{\circ} \mathrm{C}$ and there was a little difference in the optimal temperature of Ec-GlmU $\left(30^{\circ} \mathrm{C}\right)$ and Bs-GlmU $\left(37^{\circ} \mathrm{C}\right)$ (Fig. $\left.3 \mathrm{~b}\right)$. The concentration of $\mathrm{Mg}^{2+}$ had no effect on the enzymatic activity of Ec-GlmU, but Bs-GlmU activity was optimal at $15 \mathrm{mM}$ (Fig. 3c.). According to these data analysis, the huge difference about the acetyltransferase activities between Ec-GlmU and BsGlmU illustrated that both catalysis mechanisms was not identical. Further virtual amino acid mutation on Bs-GlmU was implemented to interpret these differences.

\subsection{Structural model of Bs-GlmU and virtual amino acid mutation analysis}

GlmU is considered one of the most promising antibacterial drug targets because the first reaction catalyzed by this enzyme is absent in mammals. ${ }^{48}$ E. coli and B. subtilis are two normal models for Gram-negative and Gram-positive bacteria, respectively. Studies of GlmU form E. coli and B. subtilis are extremely instructive for the development of new antimicrobial agents. The X-ray crystal structure of the Ec-GlmU acetyltransferase active site confirmed the 2-amino group of GlcN-1-PO $\mathrm{PO}_{4}$ is positioned in proximity to the AcCoA to facilitate direct attack on the thioester by a ternary complex mechanism. ${ }^{31}$ However, the mechanism of Bs-GlmU has not been reported.

The acetyltransferase active site, defined by the binding site for AcCoA, makes use of residues from all three subunits of GlmU and is positioned beneath an open cavity large enough to accommodate the Glc-1- $\mathrm{PO}_{4}$ acetyl acceptor. ${ }^{31}$ The 2-amino group of $\mathrm{GlcN}-1-\mathrm{PO}_{4}$ is positioned in proximity to AcCoA to facilitate direct attack of the thioester by a ternary complex mechanism (based on the structure of $E$. coli bifunctional GlmU). ${ }^{47}$ The Protein Data Bank (PDB) was BLAST searched for similar sequences using the target sequence (PDB ID: 3TWD-B, 3DK5-A, 2VOH-A, 3FWW-A, 1HM9-A) and the results were aligned (Fig. 4) to build a tertiary structure of monomeric BsGlmU (Fig. 5a) for homology modeling. The quality of the BsGlmU model was assessed by Ramachandran statistics ${ }^{\mathbf{4 9}}$ and Profile-3D. ${ }^{50} 93.2 \%$ of the total amino acids were located in the optimum region of the Ramachandran plot, with $3.4 \%$ in the 

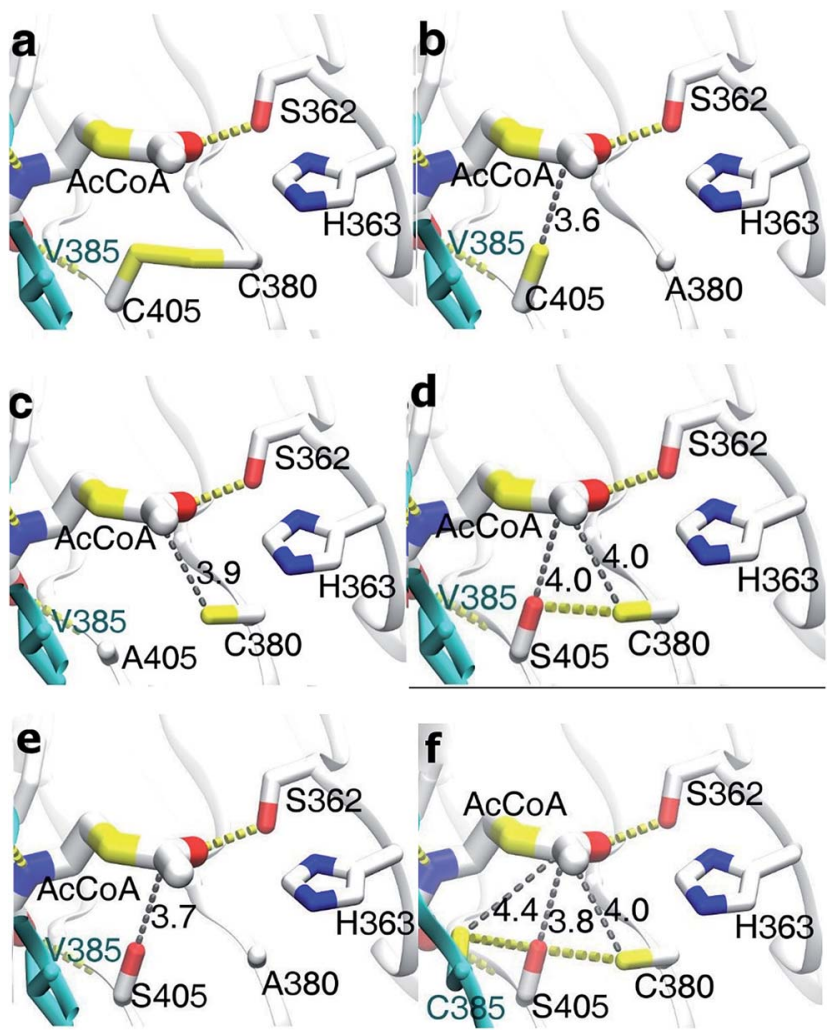

Fig. 6 Virtual amino acid mutation analysis and mutant model docking with AcCoA. $\mathrm{H}$-bonds are shown in yellow dotted lines and hydrophobic interaction is indicated as gray dotted line. Arabic numerals next to the gray dotted line represent distance of hydrophobic interaction (Å). (a) Bs-GlmU $U_{W T}$ binding AcCoA model (in this model, yellow sticks between C380 and C405 indicated the disulfide bond); (b) Bs$\mathrm{GlmU}_{\mathrm{C380A}}$ binding AcCoA model; (c) Bs-GlmU $\mathrm{C}_{405 \mathrm{~A}}$ binding $\mathrm{AcCOA}$ model; (d) Bs-GlmU $\mathrm{C} 405 S_{\text {S }}$ binding AcCoA model; (e) Bs-GlmU $\mathrm{C}_{380 \mathrm{~A}}$ c405s binding AcCoA model; (f) Bs-GlmU $U_{V 385 C-C 405 s}$ binding AcCoA model.

allowed region and $3.4 \%$ in the disallowed region (Fig. 5b). The compatibility score of the majority of the amino acids was greater than zero in the Profile-3D graph (Fig. 5c). These structure evaluation analyses showed that the model represents the Bs-GlmU structure.

The result of the sequence alignment (Fig. 4) has shown that there were several cysteine residues in acetyltransferase region of Bs-GlmU, such as C380, C405 and so on. It is wonder that the disulfide bonds between cysteine residues in this region affected the activity of the acetyltransferase. The model of BsGlmU binding a substrate AcCoA was simulated (Fig. 6a). The acetyltransferase catalytic site interacts with AcCoA through a hydrogen bond between residue S362 and carbonyl group of AcCoA and through van der Waals interactions. The close distance between C380 and C405 suggested that these two residues could form a disulfide bond. The resulting disulfide bond has a weak interaction with the carbon atom of acetyl carbonyl group of AcCoA (Fig. 6a). Moreover, the $K_{\mathrm{m}}$ value of AcCoA to Bs-GlmU $\mathrm{WT}_{\mathrm{WT}}$ was calculated (Fig. 8a) as $1.821 \mathrm{mM}$. Based on this result, it is speculated that the disulfide bond formed between $\mathrm{C} 380$ and $\mathrm{C} 405$ could seriously affect

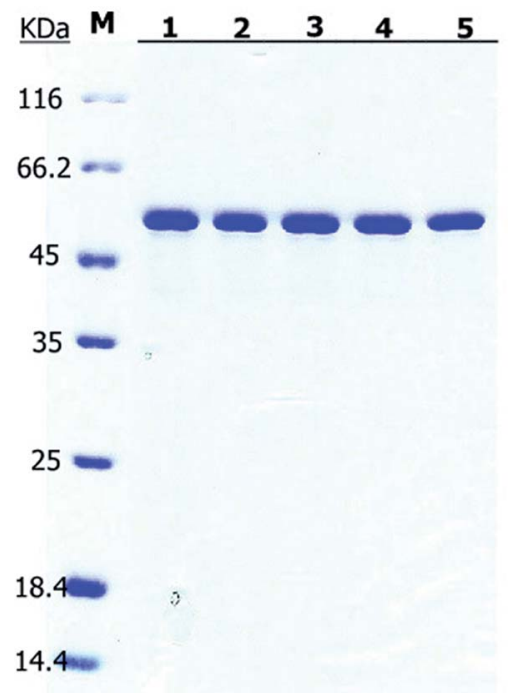

Fig. 7 SDS-PAGE of the purified Bs-GImU mutants. M: protein marker; (1) Bs-GlmU $U_{C 380 A}$; (2) Bs-GlmU $U_{C 405 s ;}$ (3) Bs-GlmU $U_{C 380 A-C 4055 ;}$ (4) Bs-

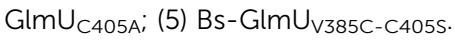

acetyltransferase of Bs-GlmU. In order to verify this speculation, the purified Bs-GlmU was dialyzed to buffer S containing DTT from $5 \mathrm{mM}$ to $10 \mathrm{mM}$ to break disulfide bonds formed by cysteine residues. The results showed that the acetyltransferase activity indeed improved in varying degrees when Bs-GlmU was dialyzed to different concentrations of DTT buffer (data not shown). It illustrated that the existence of DTT have interrupted disulfide bonds formed by cysteine residues.

Based on this pre-experimental basis, virtual amino acid mutation analysis was used to design a mutant A (Bs$\mathrm{GlmU}_{\mathrm{C} 380 \mathrm{~A}}$ ) to break the disulfide bond between C380 and C405. The Bs-GlmU ${ }_{\mathrm{C} 380 \mathrm{~A}}$ mutant binding AcCoA model shows the negative charged sulfhydryl group of C405 interacts with the acetyl carbonyl carbon of AcCoA at distance $3.6 \AA$ (Fig. 6b), which transition state is more stable than the original enzyme. Then the purified mutant protein Bs-GlmU $\mathrm{C}_{\mathrm{C} 380 \mathrm{~A}}$ was used to determine the enzyme activity and kinetic parameters (Fig. 7 and $8 \mathrm{~b}$ ). Not surprisingly, the acetyltransferase activity of Bs$\mathrm{GlmU}_{\mathrm{C} 380 \mathrm{~A}}$ was 2.6 folds high than that of Bs-GlmU $\mathrm{WT}_{\mathrm{WT}}$. Moreover, reaction time of Bs-GlmU $\mathrm{C} 380 \mathrm{~A}$ to reach equilibrium phase (from $8 \mathrm{~h}$ to $30 \mathrm{~min}$ ) reduced significantly (Table 2). The $k_{\text {cat }} / K_{\mathrm{m}}$ value of the Bs-GlmU $U_{\mathrm{C} 380 \mathrm{~A}}$ mutant increased 67.1 fold because of the disulfide bond breakdown. The $K_{\mathrm{m}}$ of AcCoA to Bs$\mathrm{GlmU}_{\mathrm{C} 380 \mathrm{~A}}$ also decreased (Table 2). All results indicated that breaking the disulfide bonds formed by C380 and C405 could obviously enhance the acetyltransferase activity.

To investigate this phenomenon, mutant $\mathrm{B}$ (Bs-GlmU $\left.\mathrm{C}_{\mathrm{C} 405 \mathrm{~A}}\right)$ was constructed. Surprisingly, the Bs-GlmU $\mathrm{C}_{\mathrm{C} 405 \mathrm{~A}}$ mutant binding AcCoA model showed that the negatively charged sulfhydryl group of C380 released in Bs-GlmU $\mathrm{C}_{\mathrm{C} 405 \mathrm{~A}}$ had a little longer distance $(3.9 \AA)$ to carbonyl carbon atom of AcCoA (Fig. 6c). This longer distance may not be able to improve stability of the transition state. It is predictable that acetyltransferase activities were not apparently increased. The kinetic 
a

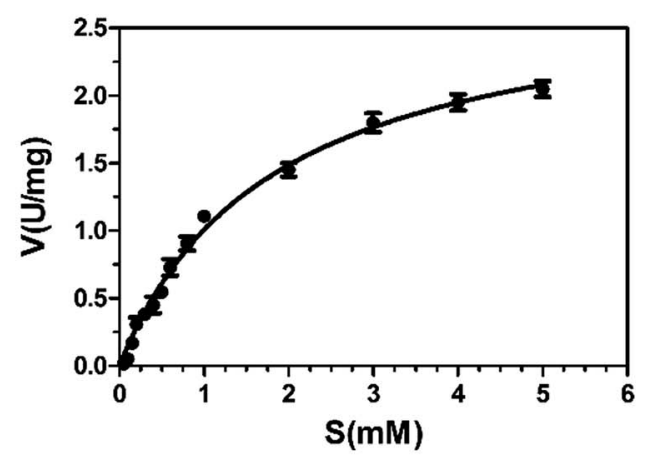

C

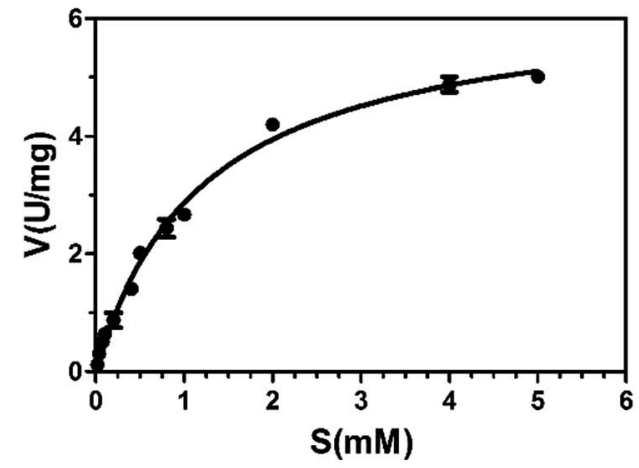

$\mathbf{e}$

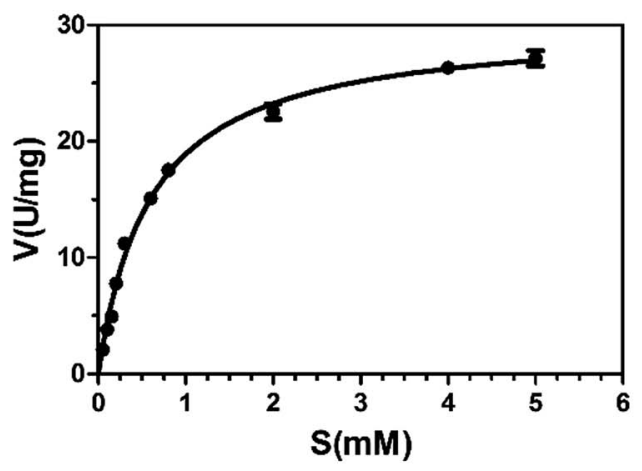

b

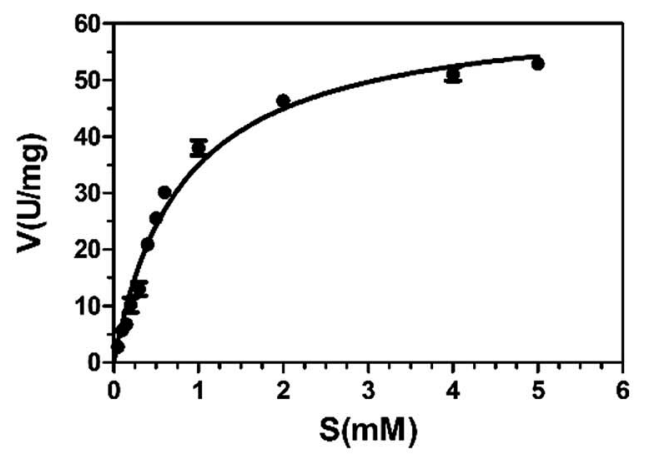

d
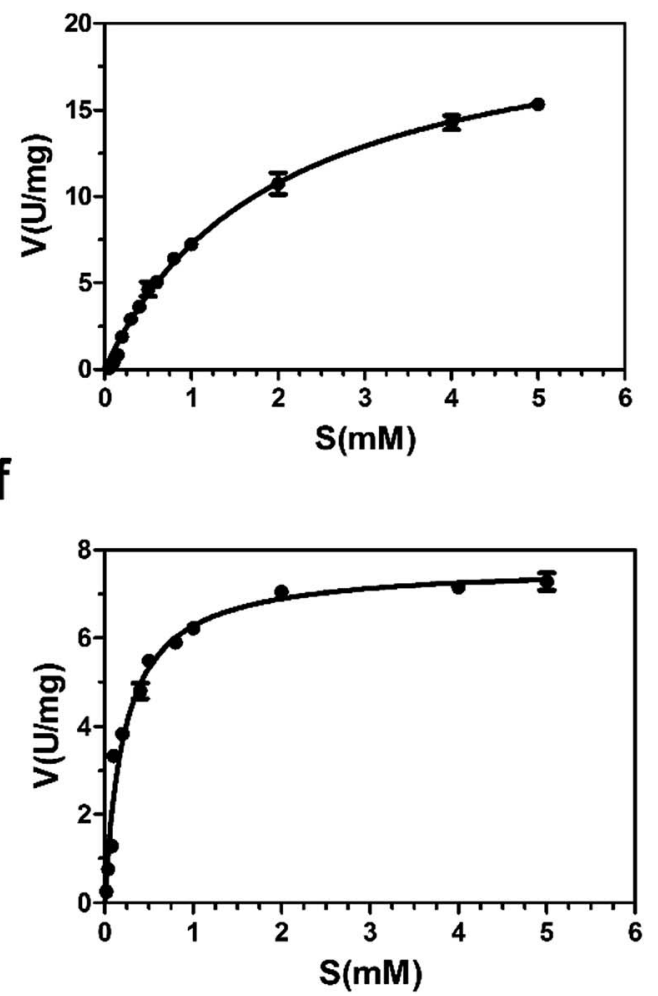

Fig. 8 Nonlinear Michaelis-Menten plots of the purified Bs-GlmU $U_{\mathrm{WT}}$ and other five mutants with AcCoA. The steady-state kinetic parameters $K_{m}$ and $V_{\max }$ for substrates AcCoA were determined at various concentrations $(0.05,0.1,0.15,0.2,0.3,0.4,0.5,0.6,0.8,1.0,2.0,4.0,5.0 \mathrm{mM})$ of AcCoA maintaining substrate GlcN1P at fixed value $(0.5 \mathrm{mM})$ under optimal reaction conditions. (a) Bs-GlmU $U_{W T}(b) B s-G l m U_{C 380 A}$; (c) Bs-

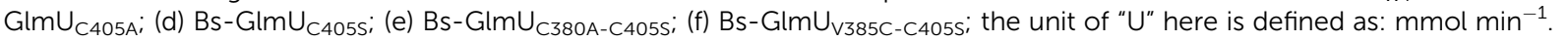

assay experiment (Fig. 8c) was also consistent with the modeling assay and showed only a slightly increase in activity $(p$ $>0.05)$. It suggested that not only the disulfide bonds formed by C380 and C405 can greatly decrease the acetyltransferase activities of Bs-GlmU, but also C405 played a key role in acetyltransferase activities of Bs-GlmU.

In order to validate this conclusion, we designed the mutant C (Bs-GlmU $\left.U_{\mathrm{C} 405 \mathrm{~S}}\right)$ which substitute cysteine with serine. Both S405 and C380 interact with the acetyl carbonyl carbon of AcCoA and increase stability of the transition state. However, the interaction force was weaker than that of the Bs-GlmU $U_{\mathrm{C} 380 \mathrm{~A}}$ mutant with a longer distance between the protein and substrate (4.0 ̊) (Fig. 6d). In addition, there was a hydrogen bond between side chains S405 and C380 that affected the stability of the transition state. In the experiment, the slightly improved stability of the transition state in Bs-GlmU $\mathrm{C}_{\mathrm{C} 405 \mathrm{~S}}$ increased slightly ( 8 fold) in the $k_{\text {cat }} / K_{\mathrm{m}}$ value (Table 2 and Fig. 8d). The residue $\mathrm{C} 405$ replaced by A or S reduced greatly the enzyme activity which demonstrates that the residue $\mathrm{C} 405$ is indeed a key amino acid in the acetyltransferase of Bs-GlmU. Additionally, a double mutant D (Bs-GlmU $\left.\mathrm{C}_{\mathrm{C} 380 \mathrm{~A}-\mathrm{C} 405 \mathrm{~S}}\right)$ was constructed. In the Bs-GlmU $U_{\mathrm{C} 380 \mathrm{~A}-\mathrm{C} 405 \mathrm{~S}}$ mutant binding AcCoA 
Table 2 The acetyl transferase kinetic parameters of BS-GlmU $U_{W T}$ and mutants

\begin{tabular}{|c|c|c|c|c|c|c|c|c|}
\hline \multirow[b]{2}{*}{ Mutants } & \multicolumn{2}{|l|}{ Properties } & \multicolumn{3}{|l|}{ GlcN-1-P } & \multicolumn{3}{|l|}{ AcCoA } \\
\hline & $\begin{array}{l}\text { Stationary } \\
\text { time }(\mathrm{h})\end{array}$ & $\begin{array}{l}\text { Optimal } \\
\text { activity (mM) }\end{array}$ & $K_{\mathrm{m}}(\mathrm{mM})$ & $k_{\text {cat }}\left(\mathrm{s}^{-1}\right)$ & $k_{\text {cat }} / K_{\mathrm{m}}\left(\mathrm{mM} \mathrm{s}^{-1}\right)$ & $K_{\mathrm{m}}(\mathrm{mM})$ & $k_{\text {cat }}\left(\mathrm{s}^{-1}\right)$ & $\begin{array}{l}k_{\mathrm{cat}} / K_{\mathrm{m}} \\
\left(\mathrm{mM} \mathrm{s}^{-1}\right)\end{array}$ \\
\hline BS-GlmU $U_{\mathrm{WT}}$ & 8 & $0.08 \pm 0.01$ & $0.333 \pm 0.012$ & $0.810 \pm 0.014$ & $2.432 \pm 1.166$ & $1.821 \pm 0.341$ & $2.459 \pm 0.241$ & $1.350 \pm 0.707$ \\
\hline BS-GlmU $U_{\mathrm{C} 380 \mathrm{~A}}$ & 0.5 & $0.21 \pm 0.02$ & $0.241 \pm 0.010$ & $25.441 \pm 0.051$ & $105.564 \pm 5.100$ & $0.603 \pm 0.068$ & $54.617 \pm 0.074$ & $90.546 \pm 1.088$ \\
\hline BS-GlmU $U_{\mathrm{C} 405 \mathrm{~S}}$ & 2 & $0.10 \pm 0.01$ & $0.359 \pm 0.014$ & $2.407 \pm 0.009$ & $6.705 \pm 0.643$ & $1.963 \pm 0.481$ & $18.512 \pm 0.472$ & $9.430 \pm 0.981$ \\
\hline BS-GlmU U $385 \mathrm{C}-\mathrm{C} 405 \mathrm{~S}$ & 6 & $0.17 \pm 0.02$ & $0.304 \pm 0.005$ & $15.756 \pm 0.008$ & $51.829 \pm 1.600$ & $0.199 \pm 0.024$ & $6.629 \pm 0.032$ & $33.262 \pm 1.333$ \\
\hline
\end{tabular}

model, neither disulfide nor hydrogen bonds formed between the 380 and 405 positions (Fig. 6e). The close distance (3.7 ̊̊) between the hydroxy group and carbonyl carbon atom of AcCoA indicated that the C380A-C405S mutation enhanced stability of transition state compared with the C405S mutation. This distance $(3.7 \AA)$ was a little longer than Bs-GlmU $\mathrm{C}_{\mathrm{C} 300 \mathrm{~A}}(3.6 \AA)$. The result of kinetic assay (Fig. 8e) was consistent with the modeling assay and showed that C380A-C405S double mutant has an increased $k_{\text {cat }} / K_{\mathrm{m}}$ value compared to Bs-GlmU $U_{\mathrm{wT}}$ (Table 2). Above results indicated that A380 could be an essential amino acid which conferred Bs-GlmU a higher acetyltransferase activity than Ec-GlmU. Finally, a comprehensive analysis conducted to all acetyltransferase activities data, this paper draws such a conclusion that C405 and A380 may act as an important role in acetyltransferase activity of Bs-GlmU.

As reported by Zhang and Olsen, C385 from one monomer of GlmU interact with $\mathrm{S} 405$ from another monomer, which could enhance the stability of the Ec-GlmU trimer. ${ }^{23,51}$ Mutant E (Bs$\left.\mathrm{GlmU}_{\mathrm{V} 385 \mathrm{C}-\mathrm{C} 405 \mathrm{~S}}\right)$ was constructed to test this interaction between amino acids. The model of Bs-GlmU $\mathrm{V}_{\mathrm{V} 385 \mathrm{C}-\mathrm{C} 405 \mathrm{~S}}$ binding AcCoA implied that C385, S405 and C380 interact with the pantetheine of AcCoA through hydrophobic interactions. The hydrogen bonds between C385-S405 and C380-S405 also enhance the stability of the transition state (Fig. 6f). The $k_{\text {cat }} / K_{\mathrm{m}}$ value of the mutant Bs-GlmU $\mathrm{V}_{\mathrm{V} 35 \mathrm{C}-\mathrm{C} 405 \mathrm{~s}}$ supported the statement (Table 2 and Fig. 8f).

\section{Conclusion}

As an essential substrate for two important biosynthetic pathways of peptidoglycan and lipid A in bacteria, studies on the GlmU enzyme has attracted increasing interests from both the industrial and the academic in recent decades. In this work, GlmU enzymes from E. coli and B. subtilis were successfully expressed and purified. Compared with Ec-GlmU and GlmU from other microorganisms, Bs-GlmU showed significantly lower acetyltransferase activity. By analyzing the result of the sequence alignment, we predicted the structure of Bs-GlmU and constructed five Bs-GlmU mutants by varying residues close to the acetyltransferase sites. The huge difference in acetyltransferase activity between mutants and Bs-GlmU $\mathrm{WT}_{\mathrm{W}}$ demonstrated that the C405 and A380 residue may play a key role in BsGlmU acetyltransferase activity. The studies on amino acid mutation in acetyltransferase of Bs-GlmU provided an important way to comprehensive understand reaction mechanism of Bs-GlmU and set up a platform for the future research on GlmU enzyme in other Gram-positive microorganisms. This work supported the development of GlmU inhibitors to potential new anti-bacterial drugs.

\section{Acknowledgements}

This work was supported by the National Natural Science Foundation of China (Grant no. 21306055), Guangdong Natural Science Foundation (Grant no. 2014A030313261), the Ph.D. Programs Foundation of Ministry of Education of China (Grant no. 20130172120041), the Fundamental Research Funds for the Central Universities (Grant no. 2015ZM161) and Special Funds for the Cultivation of Guangdong College Students' Scientific and Technological Innovation (Climbing Program Special Funds, Grant no. pdjh2015b0048). We are grateful to Xuwan Dong and Bihong Li for technical support with the clones.

\section{References}

1 G. Joanny, D. J. Le, D. Bréchemier-Baey, V. Labas, J. Vinh, P. Régnier and E. Hajnsdorf, Nucleic Acids Res., 2007, 35, 2494-2502.

2 J. S. Rush, P. D. Rick and C. J. Waechter, Glycobiology, 1997, 7, 315-322.

3 M. Bardor, R. Louvet, B. Sanit-Jean, C. Burel, B. Baiet, A. Carlier, J. P. Cadoret and P. Lerouge, EP, EP2412815, 2012.

4 M. L. Reitman, L. Lang and S. Kornfeld, Methods Enzymol., 1984, 107, 163-172.

5 S. Merello, A. J. Parodi and R. Couso, J. Biol. Chem., 1995, 270, 7281-7287.

$6 \mathrm{~J}$. Rodríguezdíaz, A. Rubiodelcampo and M. J. Yebra, Bioengineered, 2012, 3, 339-342.

7 A. H. L. Koenderman, P. L. Loppen, A. M. M. Lian and D. H. V. D. Eijnden, Biomed. Chromatogr., 1986, 1, 104-108.

8 G. L. French, Int. J. Antimicrob. Agents, 2010, 36, S3-S7.

9 I. M. Gould, Int. J. Antimicrob. Agents, 2010, 36, S1-S2.

10 R. E. Isturiz, Int. J. Antimicrob. Agents, 2010, 36(5), S19-S22.

11 R. G. Masterton, Int. J. Antimicrob. Agents, 2010, 36(5), S15S18.

12 T. R. Walsh, Int. J. Antimicrob. Agents, 2010, 36(3), S8. 
13 G. L. Moraes, G. C. Gomes, P. R. Monteiro de Sousa, C. N. Alves, T. Govender, H. G. Kruger, G. E. Maguire, G. Lamichhane and J. Lameira, Tuberculosis, 2015, 95, 95111.

14 R. Mehra, C. Rani, P. Mahajan, R. A. Vishwakarma, I. A. Khan and A. Nargotra, ACS Comb. Sci., 2016, 18(2), 100-116.

15 P. Doig, P. A. Boriacksjodin, J. Dumas, J. Hu, K. Itoh, K. Johnson, S. Kazmirski, T. Kinoshita, S. Kuroda and T. O. Sato, Bioorg. Med. Chem., 2014, 22, 6256-6269.

16 S. Wang, F. Xuan, Y. Liu, X. W. Liu, W. Lin, J. Fang and G. W. Peng, Carbohydr. Res., 2015, 413, 70-74.

17 N. Vithani and B. Prakash, Encyclopedia of Inorganic and Bioinorganic Chemistry, 2015.

18 C. Rani, R. Mehra, R. Sharma, R. Chib, P. Wazir, A. Nargotra and I. A. Khan, Tuberculosis, 2015, 95(6), 664-677.

19 H. Barreteau, A. Kovac, A. Boniface, M. Sova, S. Gobec and D. Blanot, FEMS Microbiol. Rev., 2008, 32, 168-207.

20 M. A. Pathan, A. S. Jamkhandi and A. K. S. Katti, Int. J. Pharm. Sci. Res., 2014, 5(4), 1493.

21 M. Kotnik, P. S. Anderluh and A. Prezelj, Curr. Pharm. Des., 2007, 13, 2283-2309.

22 C. R. Raetz and C. Whitfield, Biochemistry, 2002, 71, 635-700.

23 Z. Zhang, E. M. M. Bulloch, R. D. Bunker, E. N. Baker and C. J. Squire, Acta Crystallogr., 2009, 65, 275-283.

24 V. K. Singh, K. Das and K. Seshadri, PLoS One, 2012, 7, e43969.

25 J. Min, D. Lin, Q. Zhang, J. Zhang and Z. Yu, Eur. J. Med. Chem., 2012, 53, 150-158.

26 L. R. Olsen, M. W. Vetting and S. L. Roderick, Protein Sci., 2007, 16, 1230-1235.

27 D. Patin, M. Bayliss, D. Menginlecreulx, P. Oyston and D. Blanot, Arch. Microbiol., 2015, 197, 1-8.

28 R. Mehra, R. Sharma, I. A. Khan and A. Nargotra, Eur. J. Med. Chem., 2015, 92, 78-90.

29 A. M. Gehring, W. J. Lees, D. J. Mindiola, C. T. Walsh and E. D. Brown, Biochemistry, 1996, 35, 579-585.

30 R. Sharma, M. R. Lambu, U. Jamwal, C. Rani, R. Chib, P. Wazir, D. Mukherjee, A. Chaubey and I. A. Khan, J. Biomol. Screening, 2016, 21(4), 342-353.

31 L. R. Olsen, M. W. Vetting and S. L. Roderick, Protein Sci., 2007, 16, 1230-1235.
32 X. Li, C. Qi, P. Wei, L. Huang, J. Cai and Z. Xu, Prep. Biochem. Biotechnol., 2016, accepted.

33 B. Hove-Jensen, J. Bacteriol., 1992, 174, 6852-6856.

34 B. Hove-Jensen, Mol. Microbiol., 1989, 3, 1487-1492.

35 Y. Zhou, Y. Xin, S. Sha and Y. Ma, Arch. Microbiol., 2011, 193, 751-757.

36 W. Zhang, V. C. Jones, M. S. Scherman, S. Mahapatra, D. Crick, S. Bhamidi, Y. Xin, M. R. Mcneil and Y. Ma, Int. J. Biochem. Cell Biol., 2008, 40, 2560-2571.

37 J. Zhou, L. Huang, J. Lian, J. Sheng, J. Cai and Z. Xu, Biotechnol. Lett., 2010, 32, 1481-1486.

38 Y. Zhou, Y. Xin, S. Sha and Y. Ma, Arch. Microbiol., 2011, 193, 751-757.

39 Y. Zhai, M. Liang, J. Fang, X. Wang, W. Guan, X.-w. Liu, P. Wang and F. Wang, Biotechnol. Lett., 2012, 34, 1321-1326.

40 C. Meersschaert, E. Thiry and P. P. Pastoret, Rev. Elev. Med. Vet. Pays. Trop., 1988, 41, 235-242.

41 R. C. Troutman and C. Barraquer, Cornea, 1982, 1(3), 251254.

42 K. Vanommeslaeghe, E. Hatcher, C. Acharya, S. Kundu, S. Zhong, J. Shim, E. Darian, O. Guvench, P. Lopes and I. Vorobyov, J. Comput. Chem., 2010, 31, 671-690.

43 W. Humphrey, A. Dalke and K. Schulten, J. Mol. Graphics, 1996, 14, 33-38.

44 T. Unger, Y. Jacobovitch, A. Dantes, R. Bernheim and Y. Peleg, J. Struct. Biol., 2010, 172, 34.

45 d. E. F. Van and J. Löwe, J. Biochem. Biophys. Methods, 2006, 67, 67-74.

46 Y. Zhai, M. Liang, J. Fang, X. Wang, W. Guan, X. W. Liu, P. Wang and F. Wang, Biotechnol. Lett., 2012, 34, 1321-1326.

47 L. R. Olsen and S. L. Roderick, Biochemistry, 2001, 40(7), 1913-1921.

48 L. Yongmeng, Z. Yan, M. Yufang and L. Xuebing, Carbohydr. Res., 2011, 346, 1714-1720.

49 R. A. Laskowski, M. W. MacArthur, D. S. Moss and J. M. Thornton, J. Appl. Crystallogr., 1993, 26, 283-291.

50 R. Lüthy, J. U. Bowie and D. Eisenberg, Nature, 1992, 356, 8385.

51 L. R. Olsen and S. L. Roderick, Biochemistry, 2001, 40, 19131921. 